\title{
Behavioral and Brain Dynamics of Team Coordination Part II: Neurobehavioral Performance
}

\author{
E. Tognoli ${ }^{1}$, A.J. Kovacs ${ }^{1}$, B. Suutari ${ }^{1}$, D. Afergan ${ }^{2,3}$, J. Coyne ${ }^{2}$, \\ G. Gibson ${ }^{2}$, R. Stripling ${ }^{4}$, and J.A.S. Kelso ${ }^{1,5}$ \\ ${ }^{1}$ Center for Complex Systems and Brain Sciences, Florida Atlantic University, \\ Boca Raton, FL \\ ${ }^{2}$ Naval Research Laboratory, Washington, DC \\ ${ }^{3}$ Strategic Analysis Inc., Arlington, VA \\ ${ }^{4}$ Office of Naval Research, Arlington, VA \\ ${ }^{5}$ Intelligent Systems Research Center, University of Ulster, Derry, N. Ireland \\ \{tognoli, kovacs, suutari,kelso\}@ccs.fau.edu, dafergan@sainc.com, \\ \{coyne, gibson\}@itd.nrl.navy.mil, roy.stripling@navy.mil
}

\begin{abstract}
In this study, pairs of subjects performed a team-intensive task with the shared goal of clearing a virtual room from threats. The neurobehavioral dynamics of both subjects was analyzed to identify signatures of efficient team work. An ecologically valid task of room clearing was designed and a novel analysis framework was developed to address the challenge of understanding complex, continuous social processes at both behavioral and brain levels. A companion paper detailed the design of the neurobehavioral task and its associated dynamical analysis framework. In this paper, we present candidate neuromarkers for efficient room clearing and discuss key theoretical issues relating to successful team coordination.
\end{abstract}

Keywords: Neuromarkers, EEG, neurobehavioral dynamics, social behavior, complexity.

\section{Introduction}

Team coordination is critical for general human performance, and all the more when members' survival and safety depend upon efficient cooperation, such as when Marines neutralize dangers in a confined urban environment. During such tasks, a host of behavioral, cognitive and social processes have to be coordinated in space and in time in a context-dependent fashion. The goal of this study was to quantify the dynamics of neurobehavioral processes unfolding during a room clearing task that imposes high demand on both individual and team coordination. In a companion paper [1], we have presented a behavioral room clearing task. As a reminder, during this task, pairs of subjects navigate a series of rooms in a virtual environment while executing formal behavioral and mental operations that take place during real room clearing performance: stacking, tap signaling, entry, orientation to corner of domination, pieing, friend/enemy detection, shoot-no shoot decision making and restacking. The task requires the recruitment of sociocognitive, perceptual and 
attentional processes that must be coordinated within and across the brains of both team members. The goal of this study was to decipher the neurobehavioral organization of these processes from continuous dual-EEG recordings. For details on the task and analysis, please refer to [1]. In the following, we present neurobehavioral results obtained from a group of 18 subjects ( 9 pairs). Further, from the theory of Coordination Dynamics [2], we discuss key theoretical issues that shed light on successful team coordination.

\section{Neurobehavioral Performance}

Examples of behavioral trajectories for complete building clearing are illustrated in Figure 1. A spatial map is shown in (A) and corresponding temporal courses (avatars' rate of movement) in (B). Subjects complied sufficiently well with training instructions to yield efficient behavioral performance for most pairs. They generally performed well-phased series of motion and gaze behavior (Figure 1B).

For each room, motion was typically divided into two cleanly separated phases (entry-to-corner and restacking). Occasionally, team members lost coordination (not shown). In this case, it was generally observed that coordination was not reinstated before the onset of the subsequent building, suggesting that in our novice teams, behavioral flows are more strongly determined by the time scale of the building, than that of the rooms. To remediate such detrimental performance, resetting would be required at shorter time scales: during restacking (time scale of room) and immediately after the onset of disorganized behavior (time scale of instantaneous behavior).

Analysis of movement coordination also suggested that novice subjects were not necessarily weakly coupled: a large number of low-performance trials (variable "time to clear room") showed that subjects' movements were strongly determined by their partners': for instance, one avatar initiating a movement only when the partner initiates (in-phase coordination) or terminates his movements (anti-phase coordination). In contrast, in time-effective trials, subjects were able to phase movements more flexibility, exhibiting a coordination pattern with tendencies for the avatars to come together at the same time as to maintain a degree of individual autonomy. Coordination dynamics dubs this complementary tendency metastability [2-4]. Metastable forms of coordination have been shown to enhance complexity $[5,6]$. Recent work in our laboratory suggests that several neural mechanisms exist to dissolve coordinated behavior. One is increased activity in phi1, the first component of the so-called 'phi complex'--a neuromarker of social coordination and lack thereof

[7]. Another is an instantaneous neuromarker of behavioral segregation which is observed in steady-state social coordination at the transition from coordinated to uncoordinated behavior [8]. Interestingly, at the transition to independent behavior, the brains of interacting subjects are strongly coupled by a mechanism called "synchronized brain transitions" [8-9]. In fact, between-brain coupling is as strong at the transition to independent behavior as it is at the onset of coordinated behavior. This suggests that efficient independent behavior is achieved by virtue of exchange of information and coupling of the subjects' brains, in agreement with the theory of metastability [2-4]. 


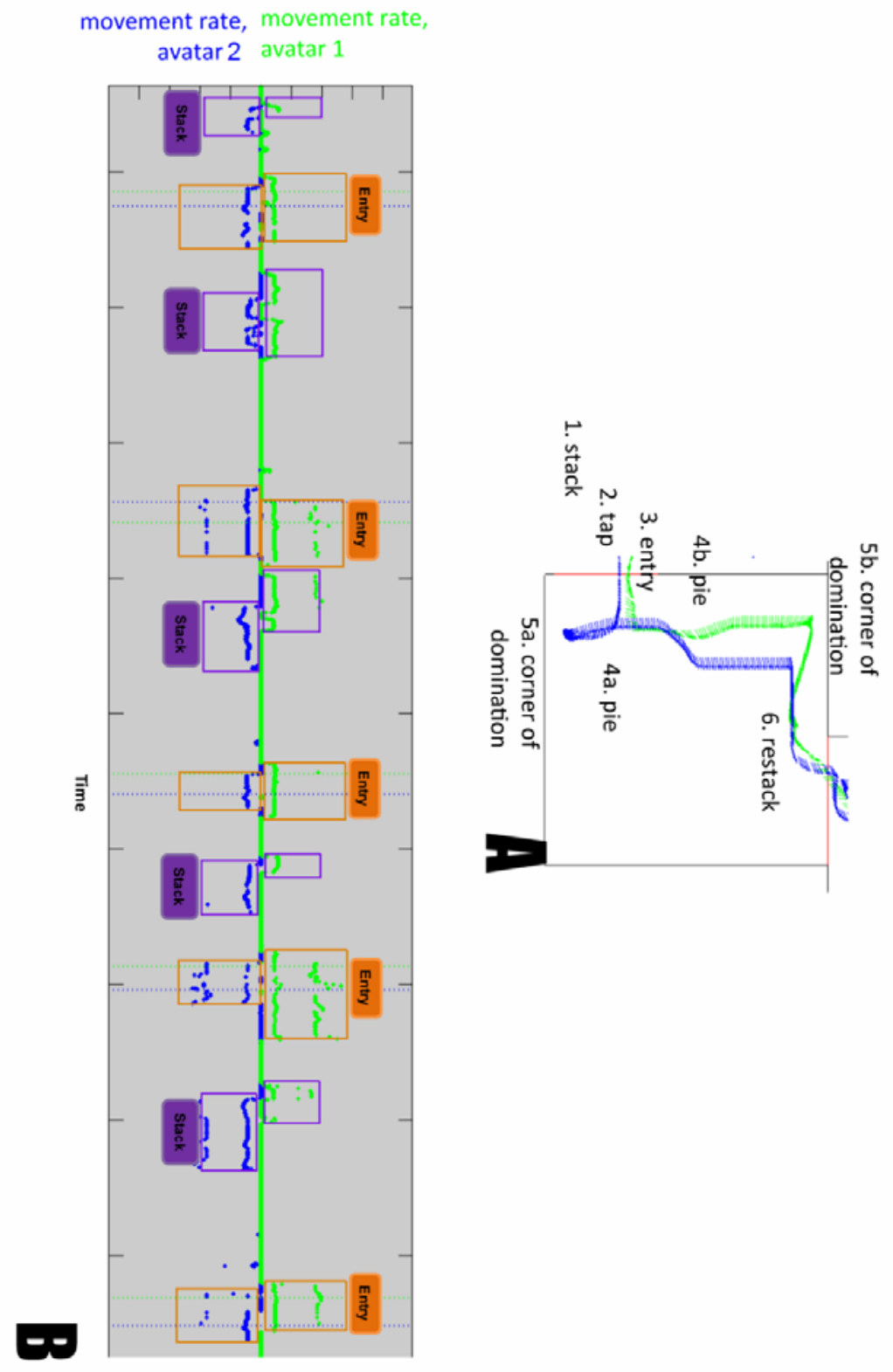

Fig. 1. Spatial view of the green and blue avatar paths in a room with the behavioral markers of interest annotated (A). Corresponding temporal view of the rate of change of movement for the entire building (B) for well-executed room clearing. 

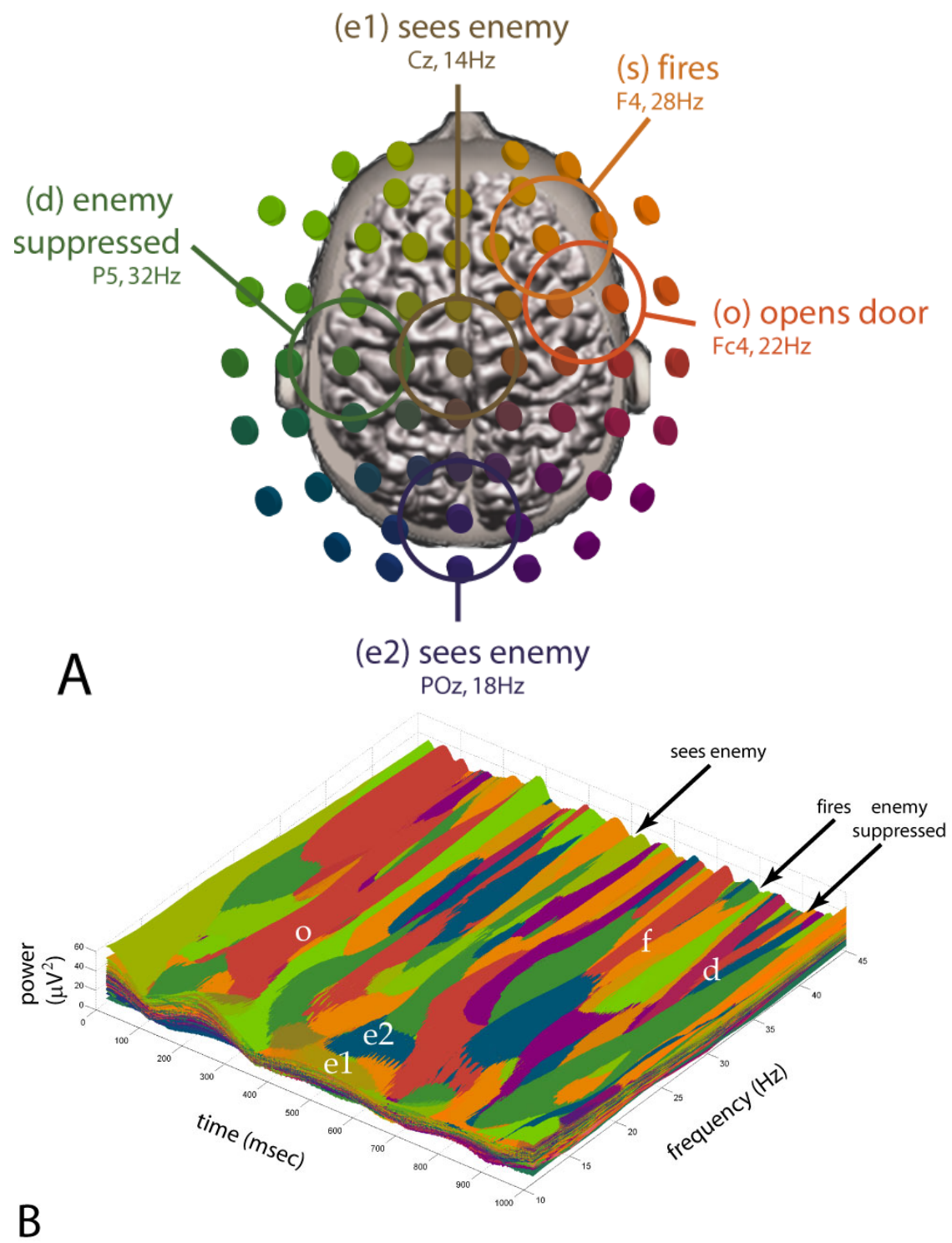

Fig. 2. Candidate neuromarkers for a variety of behaviors observed after entry in a hostile room, shown with their typical topography and frequency (A) along with their time distribution as observed from time-frequency-power plots (B). The colors in (B) reflect the spatial distribution of power as illustrated in (A). In this single trial (B), $1000 \mathrm{~ms}$ of EEG data are displayed, with the agent uncovering the enemy avatar and firing in less than $300 \mathrm{msec}$.

In previous studies, we identified several neuromarkers of social $[7,10]$ and attentional behavior $[7,11]$ in the $10 \mathrm{~Hz}$ EEG frequency band. In the virtual environment created for the present study, performance of room clearing ("time to clear room") was bounded by experimental parameters (e.g. maximal avatar velocity, room dimension). 
Execution of all behavioral, cognitive and social processes that led to task completion (see section 2.2 from companion paper [1]) was typically performed in 2 or 3 seconds, a time scale too short for EEG's slow frequencies alone to be useful information carriers. As a consequence, we extended our analysis to the beta and gamma frequency ranges $(14-30 \mathrm{~Hz}$ and $30-60 \mathrm{~Hz}$ respectively). Analysis was conducted at three time scales: at the level of instantaneous behavior using either spatio-spectral or spatio-temporal techniques (neuromarkers type I); for durations of time that match the execution of goal-driven behaviors (e.g. reaching a corner of domination: neuromarkers type II); for periods extending over completion of the task (neuromarkers type III). Analysis suggests candidate neuromarkers for a number of individual and social behaviors (see e.g., type I neuromarkers, Figure 2A). These neuromarkers can readily be seen in a single trial from the spatio-temporo-spectral graphs (Figure 2B). Because of the covariation of many behavioral, cognitive and social processes during task performance, further work is ongoing to assess the specificity and reliability of candidate neuromarkers. This further work is guided by the relevant time scales of behavioral processes, and their correspondence with the duration of neural processes (Figure 2B).

Neuromarkers of type II-III emphasize the importance of time scales for team coordination. For instance, we identified a beta-gamma complex over the left rolandic region appearing while leaders moved toward the corners of domination. These neural complexes appeared only in well-coordinated trials (best half of 'coordinated entry' variable, see section 2.6 from companion paper [1]), and we did not detect any particular behavior that was immediately served by such neural patterns.

We hypothesize that the beta-gamma complex that occurs during the $5^{\text {th }}$ behavioral phase of room clearing (see figure 1A) is a remnant of earlier behavior during the $3^{\text {rd }}$ (entry) phase. Such diachronic phenomena that emerge across multiple frequency bands in the brains of team members illustrate the multiple time scales that have to be considered in explaining realistic social behavior (Figure 2B). Even though preliminary, the present data argue against two time-worn features of classical neuroscience paradigms: impoverished contexts for task performance ("all other things being equal") and short, iterative, experimenter-controlled stimulus-response manipulations. In contrast, the ecologically valid task that we have developed retains the essence of the real situation and is already capturing meaningful neurobehavioral signatures of brain self-organization during team work.

\section{Concluding Remarks}

The notion that individuals often cooperate in a reciprocal and synergistic way to achieve a common goal -joint action as it is sometimes called- is currently a topic of much interest in social neuroscience. The idea can be traced back to efforts aimed at identifying functional synergies in complex human movements that require the coordination of many degrees of freedom [12]. Here we have presented preliminary findings in an ecologically valid task aimed at understanding the coordinated action of two team members committed to accomplishing a common goal. To accommodate for emergent complexity, both in terms of the many processes to be coordinated and the multiple frequencies on which they occur, both neurally and behaviorally, we 
developed a novel framework that rests on the combined analysis of, and intimate relation between, behavioral dynamics and real-time, continuous EEG.

Crucially, such a framework is necessary to track the emergence of neurobehavioral processes on multiple space and time scales. This, in turn, allows team work to persist both during behavioral phases of strong informational exchange (such as entry), and during independent phases (such as reaching the corner of domination). In the absence of direct informational exchange, social coordination can be accomplished by virtue of memory processes. In his classic work "The Strategy of Conflict" [13], the Nobel Laureate Thomas Schelling describes experiments in which people coordinate their future behavior by virtue of shared knowledge, even though there is no interaction whatsoever between the individuals. We hypothesize that such phenomena are important components in the training of coordinated teams. We have previously shown that individual behavior can be modified following mutual information exchange even though the source of coupling is no longer available: a persistence of 'social memory' in individual agents [14]. We have also shown that sensorimotor (mu) rhythms of the human brain are modulated by memory requirements imposed on leaders and followers in social imitation tasks [10]. The present results on team coordination reveal diachronic neurobehavioral processes during coordinated room clearing (see section 2). The outlook is exciting for determining how such neuromarkers can be used to predict team performance in real time and thereby improve training.

Acknowledgments. The technical support of William McLean is acknowledged. This work is supported by the US Office of Naval Research Contract N000140510117. JASK and ET are also supported by NIMH Grant MH080838, NSF Grant BCS0826897and the Davimos Family Endowment for Excellence in Science.

\section{References}

1. Tognoli, E., Kovacs, A.J., Suutari, B., Afergan, D., Coyne, J., Gibson, G., Stripling, R., Kelso, J.A.S.: Behavioral and brain dynamics of team coordination part I: task design (this issue)

2. Kelso, J.A.S.: Dynamic patterns: the self-organization of brain and behavior. The MIT Press, Cambridge (1995)

3. Tognoli, E., Kelso, J.A.S.: Brain coordination dynamics: True and false faces of phase synchrony and metastability. Progress in Neurobiology 87, 31-40 (2009)

4. Kelso, J.A.S., Tognoli, E.: Toward a complementary neuroscience: metastable coordination dynamics of the brain. In: Kozma, R., Perlovsky, L. (eds.) Neurodynamics of Cognition and Consciousness, pp. 39-59. Springer, Berlin (2007)

5. Friston, K.J.: Transients, metastability, and neuronal dynamics. Neuroimage 5, 164-171 (1997)

6. Sporns, O.: Complex neural dynamics. In: Jirsa, V.K., Kelso, J.A.S. (eds.) Coordination Dynamics: Issues and Trends, pp. 197-215. Springer, Berlin (2004)

7. Tognoli, E., Lagarde, J., DeGuzman, G.C., Kelso, J.A.S.: The phi complex as a neuromarker of human social coordination. Proc. Natl. Acad. Sci. USA 104, 8190-8195 (2007) 
8. Benites, D., Tognoli, E., DeGuzman, G.C., Kelso, J.A.S.: Brain coordination dynamics: Continuous EEG tracking of the neural functional organization in a social task. Psychophysiology 47, S75-S75 (2010)

9. Tognoli, E., DeGuzman, G.C., Kelso, J.A.S.: Interacting humans and the dynamics of their social brains. In: Wang, R., Gu, F. (eds.) Advances in Cognitive Neurodynamics (II), pp. 139-143. Springer, Heidelberg (2010)

10. Suutari, B., Weisberg, S., Tognoli, E., Kelso, J.A.S.: Neuromarkers of individual and social Behavior (submitted)

11. Calderon, R.: Brain computer interface and neuroprosthetics (Master's Thesis), Florida Atlantic University (2008)

12. Kelso, J.A.S.: The nature of human interlimb coordination. Science 203, 1029-1031 (1979)

13. Schelling, T.: Strategy of conflict, 2nd edn. Harvard University, London (1980)

14. Oullier, O., De Guzman, G.C., Jantzen, K.J., Lagarde, J., Kelso, J.A.S.: Social coordination dynamics: Measuring human bonding. Social Neuroscience 3, 178-192 (2008) 